\title{
Old but Ageless Disease: Neurosyphilis with Ocular Manifestations in an HIV-Negative Patient
}

\author{
Süda Tekin¹ (D), Poyraz Düzgün², Merih Oray³, ilknur Tuğal-Tutkun³ (DD \\ 1 Department of Infectious Diseases and Clinical Microbiology, Koç University School of Medicine, İstanbul, Turkey \\ 2 Koç University School of Medicine, İstanbul, Turkey \\ 3 Department of Opthalmology, Istanbul University, Istanbul School of Medicine, Istanbul, Turkey
}

\begin{abstract}
Syphilitic uveitis is mostly observed in patients with comorbid risk factors such as human immunodeficiency virus (HIV). Here, we present a case of syphilitic uveitis that began with painless oral ulcers in an HIV negative male, whose primary and secondary phases were not detected.

Case: A 47-year old male with no significant medical history arrived at the Ear-Nose-Throat Department in September 2018 with a sore throat, fatigue, fever, and painless ulcers in his mouth. After a while, he applied to the Ophthalmology Department with complaints of stinging and decreased vision in the left eye. Hyperotofluorescent foci of his right eye were active in the upper and temporal parts of the macula. He was then diagnosed with syphilitic uveitis in our unit and was found HIV-negative. His test results showed positive of Venereal Diseases Research Laboratory (VDRL): 1/64 and T. pallidum haemagglutination assay: 1/10240. Lumber punction was performed with no cells/microorganisms. His cerebrospinal fluid investigation revealed positive VDRL of 1:32 titre. He was successfully treated with crystallized penicillin $G$ and topical corticosteroids on his eyes. On the $14^{\text {th }}$ day of the treatment, the patient was discharged with no complaints with his eyes. Syphilis should always be kept in mind -especially in low-risk groups such as those HIV-negative - in the differential diagnosis of painless ulcers and ocular manifestations even if the lesion sites are atypical in order to prevent delay in diagnosis and treatment. Keywords: Neurosyphilis, ocular manifestation, HIV negative.
\end{abstract}

\section{INTRODUCTION}

S yphilis is a sexually transmitted disease (STD) caused by Treponema pallidum (1) Primary and secondary syphilis increased to a rate of 5.3 cases per 100000 in 2013 from the rate of 2.1 per 100000 in 2000 (2). Neurosyphilis is the most feared and severe complication of syphilis and may occur at any time after the initial infection. Uveitis accounts for the majority of cases of ocular syphilis and is observed in 0.6-2.0\% of patients with syphilis $(3,4)$. Syphilitic uveitis is seen mostly in patients with comorbidities, including HIV infection $(5,6)$. Here, we present a case of syphilitic uveitis be-

\section{Corresponding Author: Süda Tekin \\ E-mail: suda.tekin@gmail.com}

Received: May 26, 2020 Accepted: July 18, 2020 Published: November 20, 2020

\section{Suggested citation:}

Tekin S, Düzgün P, Oray M, Tuğal-Tutkun i. Old but Ageless Disease: Neurosyphilis with Ocular Manifestations in an HIV Negative Patient. Infect Dis Clin Microbiol 2020; 3: 163-167.

DOI: 10.36519/idcm.2020.0014 

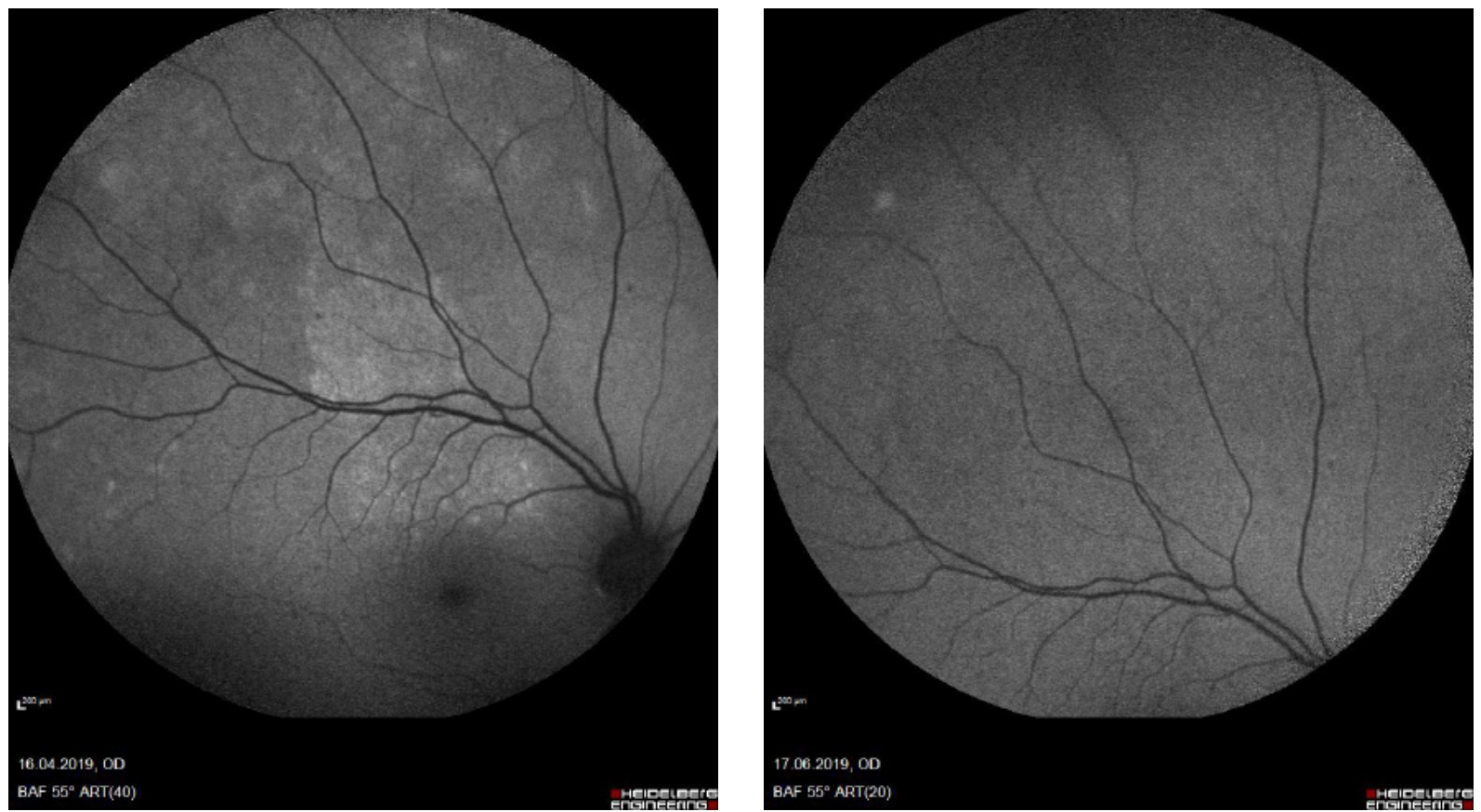

\section{Figure 1.}
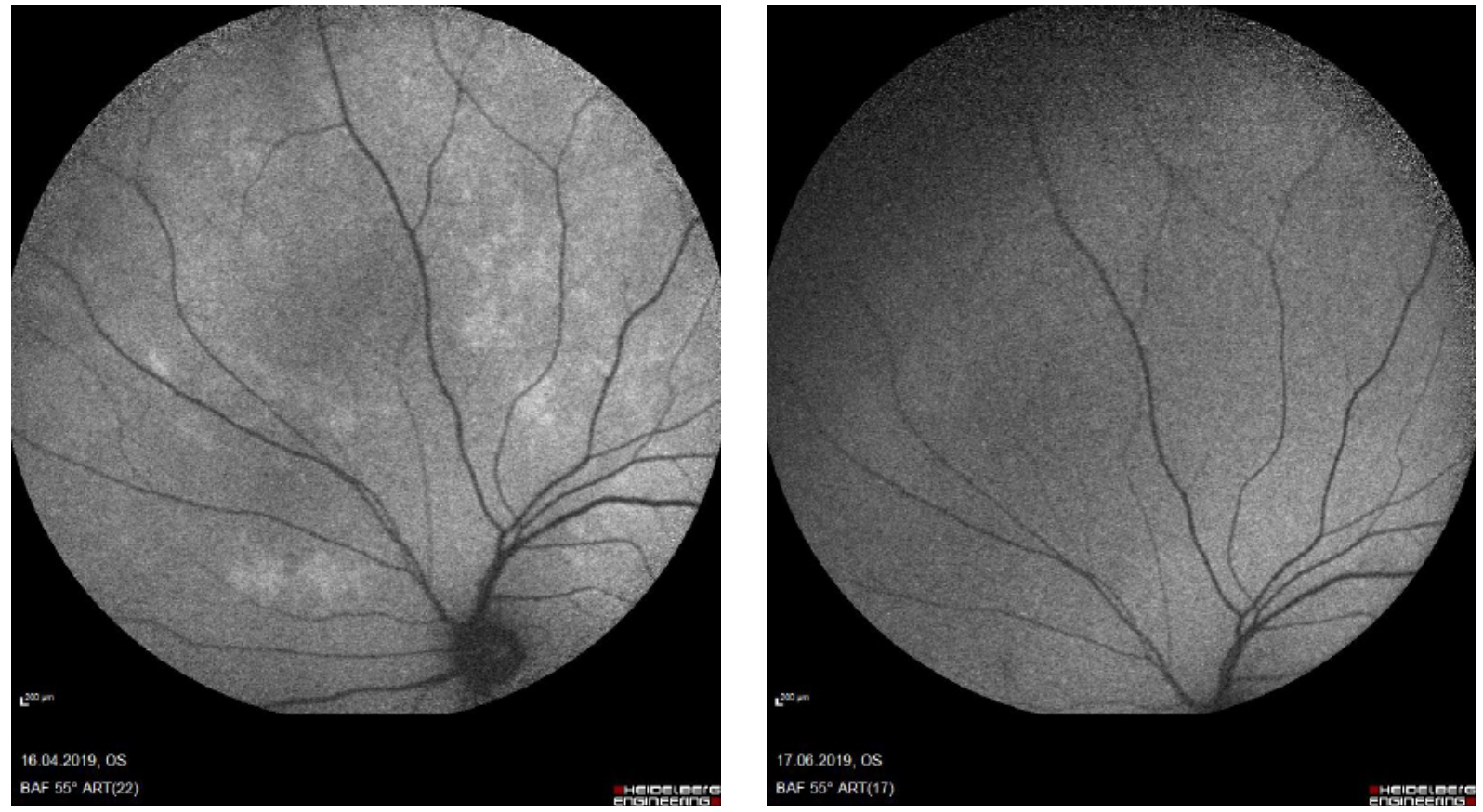

Figure 2.

ginning with the painless oral ulcers in an HIV negative male whose primary and secondary phases were not detected.

\section{Case}

A 47-year old male with no significant medical history arrived at the Ear-Nose-Throat (ENT) Department in September 2018 with a sore throat, fatigue, 
fever, and painless ulcers in his mouth. Biopsy of the lesions showed the infiltration of plasma cells. He did not receive additional treatment. In April 2019, he applied to the Ophthalmology Department, complaining of stinging and decreased vision in the left eye. The image of the right eye with a fundus autofluorescence is shown in Figure 1. Hyperotofluorescent foci were active in the upper and temporal parts of the macula before therapy. His symptoms were evaluated to indicate uveitis, and his blood investigation revealed positive on Venereal Diseases Research Laboratory (VDRL) test: 1/64. Therefore he was directed to our Infectious Diseases and Clinical Microbiology Department. After hospital admission, full blood count did not show any sign of infection, with a white cell count of $7.9 \times 109 / \mathrm{L}$. The patient had positive results on VDRL test (1/64) and Treponema pallidum haemagglutination assay (1/10240). Lumbar puncture was performed with no cells/microorganisms. His cerebrospinal fluid (CSF) investigation revealed a positive VDRL test of $1 / 32$ titre. He was found HIV-negative. He was then diagnosed with syphilitic uveitis as a complication of neurosyphilis. He was treated with $24 \mathrm{MU}$ of aqueous crystal penicillin $G$ per day, administered as $4 \mathrm{MU}$ intravenously over four hours for 14 days, and topical corticosteroids on his eyes three times two drops. An ophthalmological re-evaluation revealed findings consistent with ocular syphilis. On the 14th day of the treatment, the patient had no complaints with his eyes (Figure 2). Due to ocular symptoms, topical corticosteroids are administered while under penicillin treatment. During admission, his symptoms regressed, and he had no additional symptoms. After a compilation of 14 days of IV penicillin $G$ treatment, he was discharged. He then continued ophthalmology and infectious diseases follow-up. He did not have any remaining symptoms.

\section{DISCUSSION}

In this case report, a syphilitic uveitis case, one of the most feared and severe complications of syphilis, beginning with the painless oral ulcers in an HIV negative male, is discussed.

Syphilis is a sexually transmitted infection caused by Treponema pallidum. Before the antibiotic era, studies were relatively more explicit about identi- fying stages and progression of untreated syphilis. Despite the knowledge and preventive measures, the World Health Organization (WHO) estimates that the number of cases is steadily increasing (7). Neurosyphilis cases are now mainly associated with HIV patients and men who have sex with men $(5,6)$. Twenty-five to 35 percent of syphilis patients who develop neurosyphilis are asymptomatic (8).

Although the main transmission route is sexual, $\mathrm{T}$. pallidum can also cross the placental barrier. Open lesions are necessary for sexual transmissions, such as primary chancre and condyloma lata. Infectious courses may be divided into two categories as early and late syphilis. The early-stage contains the primary and secondary infection. Primary infections are characterized by chancres, while secondary infections can be disseminated into various organs and systems. Late syphilis is usually used for tertiary infection, and major causes of mortality are cardiovascular and central nervous system involvement.

In our case, the presentation and course of the infection are quite uncommon. Based on history, the patient admits to an ENT clinic with painless ulcers. Lesions of the oral cavity are diverse, and establishing a solid differential diagnosis may be challenging. Oral mucosal lesions may be roughly divided into five categories as normal variants of the mucosa, red/white lesions, pigmented lesions, ulcerative/eruptive lesions, and miscellaneous lesions. As a priority, a physician mostly tries to rule out malignant and premalignant lesions (9). In this case, the most significant feature of the lesion is that it is a painless ulcer. Ulcerative lesions in the oral cavity can be categorized as aphthous ulcerations, infections, and a manifestation of immune-related diseases, drug-induced, or other lesions. Multiple painless ulcers in the genital area may quickly arouse suspicion toward syphilis, but one may also expect for primary lesions first to appear on the oral mucosa, depending on the route of transmission. Taking a biopsy is also a safe and valid option when a diagnosis cannot be made with history and gross examination. Granuloma with plasma cell infiltration is typical pathologic finding for syphilis, wherever the site of lesion is (10). However, in our case, pathology only reported "plasma cell infiltra- 
tion", without indicating a granuloma formation. Although abnormal, a pathological finding may be overlooked, hence the diagnosis and the treatment may be delayed.

Months later, our patient was admitted to an ophthalmology clinic complaining from stinging and vision loss in the left eye. He was diagnosed with uveitis and then was referred to our clinic with a suspicion of syphilis. It is quite essential to acknowledge that uveitis secondary to neurosyphilis has a distinct pattern that can be recognized by an experienced ophthalmologist (11). In case that the etiology of uveitis was not identified, and steroids were used (as in the majority of uveitis cases), the progression of central nervous system (CNS) in volvement may be augmented. It is also crucial to interpreting the signs combined with patient history.

After diagnosed with neurosyphilis due to ocular manifestations, evaluating the involvement of other CNS structures (meninges, vasculature, parenchyma) is rather intriguing. Clinical evaluation and spinal fluid examination consist of the basis of diagnosis (12). Non-treponemal and treponemal tests from CSF hold great value. In our case, CSF findings also showed positivity for syphilis and without any indication of another CNS pathology. Ruling out other sexually transmitted diseases are also necessary for every case with STDs. We applied a 14 day of crystalline penicillin G 24 million units per day, while the guidelines suggest 10-14 days (13). Even debated still, we also applied benzathine penicillin 2.4 million units IM, just before discharge. Follow up visits are also planned for infectious diseases and ophthalmology departments. We also planned a control serological test from serum and CSF.

\section{CONCLUSIONS}

This case highlights the importance of considering syphilis on the differential diagnosis of painless ulcers, even when the lesion site is atypical. The oral lesions should be judged carefully based on patient history and features of lesions. Biopsy should not be trusted solely as errors in interpretation are not uncommon. Ophthalmological findings are rather distinctive and may help to establish a diagnosis. Appropriate diagnosis and treatment are key elements to prevent syphilis from a more morbid course.
Informed Consent: Written consent was obtained from the patient.

Peer-review: Externally peer-reviewed

Author Contributions: Concept - S.T., P.D., M.O.; Design - S.T., P.D.; Supervision - S.T., P.D.; Fundings - S.T.; Materials - ALL; Data Collection and/or Processing - S.T.; Analysis and/or Interpretation - S.T., P.D., İ.T.T.; Literature Review - S.T.; Writer - S.T.; Critical Reviews - S.T., P.D., İ.T.T.
Conflict of Interest: The authors have no conflict of interest to declare.

Financial Disclosure: The authors declared that this study has received no financial support

\section{REFERENCES}

1 Larsen SA. Syphilis. Clinic Lab Med 1989; 9: 545-57.

2 Patton ME, Su JR, Nelson R, Weinstock H. Centers for Disease Control and Prevention (CDC). Primary and secondary syphilis--United States, 2005-2013. MMWR Morb Mortal Wkly Rep 2014; 63: 402-6.

3 Balba GP, Kumar PN, James AN, Malani A, Palestine AG, Welch JN, et al. Ocular syphilis in HIV-positive patients receiving highly active antiretroviral therapy. Am J Med 2006; 119: 448 e21-448.e4.48E25.
4 Mathew RG, Goh BT, Westcott MC. British Ocular Syphilis Study (BOSS): 2-year national surveillance study of intraocular inflammation secondary to ocular syphilis. Invest Ophthalmol Vis Sci 2014; 55: 5394-400.

5 Albini T, Callaway NF, Pershing S, Wang SK, Moshfeghi AA, Moshfeghi DM. Trends in hospitalization and incidence rate for syphilitic uveitis in the United States from 1998 to 2009. Am J Ophthal 2017; 180: 133-41.

6 Abara WE, Hess KL, Neblett Fanfair R, Bernstein KT, Paz-Bai- 
ley G. Syphilis trends among men who have sex with men in the United States and western Europe: a systematic review of trend studies published between 2004 and 2015. PLoS One 2016; 11: e0159309.

7 WHO. Report on global sexually transmitted infection surveillance 2018. Available from: URL: http://www.who.int/reproductivehealth/publications/stis-surveillance-2018/en/

8 Yanhua W, Haishan S, Le H, Xiaomei Z, Xinru C, Ling L, et al. Clinical and neuropsychological characteristics of general paresis misdiagnosed as primary psychiatric disease. BMC Psychiatry 2016, 16:1-6.
9 9Madani, M, Berardi T, Stoopler ET. Anatomic and examination considerations of the oral cavity. Med Clin North Am 2014; 98:1225-38.

10 Engelkens HJ, ten Kate FJ, Vuzevski VD, van der Sluis JJ, Stolz E. Primary and secondary syphilis: A histopathological study. Int J STD \& AIDS 1991; 2(4): 280-284. https://doi.org/10.1177\% 2F095646249100200411. 